Internat. J. Math. \& Math. Sci.

Vol. 22, No. 3 (1999) 579-586

S 0161-1712<99>22579-3

(c) Electronic Publishing House

\title{
LEVEL CROSSINGS AND TURNING POINTS OF RANDOM HYPERBOLIC POLYNOMIALS
}

\author{
K. FARAHMAND and P. HANNIGAN
}

(Received 1 October 1997)

\begin{abstract}
In this paper, we show that the asymptotic estimate for the expected number of $K$-level crossings of a random hyperbolic polynomial $a_{1} \sinh x+a_{2} \sinh 2 x+\cdots+$ $a_{n} \sinh n x$, where $a_{j}(j=1,2, \ldots, n)$ are independent normally distributed random variables with mean zero and variance one, is $(1 / \pi) \log n$. This result is true for all $K$ independent of $x$, provided $K \equiv K_{n}=O(\sqrt{n})$. It is also shown that the asymptotic estimate of the expected number of turning points for the random polynomial $a_{1} \cosh x+a_{2} \cosh 2 x$ $+\cdots+a_{n} \cosh n x$, with $a_{j}(j=1,2, \ldots, n)$ as before, is also $(1 / \pi) \log n$.
\end{abstract}

Keywords and phrases. Gaussian process, number of real roots, Kac-Rice formula, normal density, covariance matrix.

1991 Mathematics Subject Classification. Primary 60H99; Secondary 42BXX.

1. Introduction. Let

and

$$
T_{1}(x)=\sum_{j=1}^{n} a_{j} \cosh j x
$$

$$
T_{2}(x)=\sum_{j=1}^{n} a_{j} \sinh j x,
$$

where $a_{1}, a_{2}, \ldots, a_{n}$ is a sequence of independent normally distributed random variables with mean zero and variance one. Let $N_{K}^{(i)}(\alpha, \beta)$ be the number of real roots of the equation $T_{i}(x)-K=0$ and $M^{(i)}(\alpha, \beta)$ be the number of real roots of $T_{i}^{\prime}(x)$. In both cases, the interval is $(\alpha, \beta)$ and $i=1,2$. Clearly, $M^{(i)}(\alpha, \beta)$ represents the number of turning points of $T_{i}(x)$ on the interval $(\alpha, \beta)$. It proves convenient to denote the expected number of real roots of $T_{i}(x)-K$ and $T_{i}^{\prime}(x)$, on the interval $(\alpha, \beta)$, by $E N_{K}^{(i)}(\alpha, \beta)$ and $E M^{(i)}(\alpha, \beta)$, respectively. Bharucha-Reid and Sambandham [1] reported an unpublished result of Das [2], where it is stated that for $K=0, E N_{0}^{(1)}(-\infty, \infty)$ $\sim(1 / \pi) \log n$, the random coefficients being the same as in (1.1). Farahmand [4] obtained the same asymptotic value as Das for $E N_{K}^{(1)}(-\infty, \infty)$, where $K=O(\sqrt{n})$. Farahmand highlighted the surprising way the hyperbolic polynomial mimics certain characteristics of both the trigonometric and the algebraic polynomials. In a more recent work, Farahmand [5] showed that $E M^{(2)}(-\infty, \infty) \sim(1 / \pi) \log n$, where the random coefficients are again those outlined in (1.1) and (1.2). Clearly, there remains two unsolved complementary problems, that is $E M^{(1)}(-\infty, \infty)$ and $E N_{K}^{(2)}(-\infty, \infty)$. These problems are the subject of this paper. We prove the following theorems. 
THEOREM 1. If the coefficients of $T_{2}(x)$ in (1.2) are independent normally distributed random variables with mean zero and variance one and $K_{n} \equiv K$ such that $K^{2} /(n \log n)$ tends to zero as $n$ tends to infinity, then

$$
E N_{K}^{(2)}(-\infty, \infty) \sim\left(\frac{1}{\pi}\right) \log n .
$$

THEOREM 2. If the coefficients of $T_{1}(x)$ in (1.1) are independent normally distributed random variables with mean zero and variance one and $n$ tends to infinity, then

$$
E M^{(1)}(-\infty, \infty) \sim\left(\frac{1}{\pi}\right) \log n .
$$

These results may not have been derived until now because of the difficulty in applying the conventional Rice formula to both of these cases near zero. In our method, we make the unusual step of determining the expected number of roots for the polynomial's derivative to simplify the analysis. More comprehensive results are known for the random algebraic polynomial $\sum_{j=1}^{n} a_{j} x^{j}$. We refer the reader to the pioneering works of Littlewood and Offord [8, 7] and the more recent works of Wilkins [11] and Offord [9].

2. Level crossings and turning points formulae. We employ the extension of a formula obtained by Rice [10] given by Farahmand [3], where $\operatorname{erf}(x)=\int_{0}^{x} \exp \left(-t^{2}\right) d t$, that is

$$
\begin{aligned}
E N_{k}^{(i)}(\alpha, \beta)= & \int_{\alpha}^{\beta}\left(\frac{\Delta_{i}}{\pi A_{i}^{2}}\right) \exp \left(\frac{-B_{i}^{2} K^{2}}{2 \Delta_{i}^{2}}\right) d x \\
& +\int_{\alpha}^{\beta}\left(\frac{\sqrt{2}}{\pi}\right) K D_{i} A_{i}^{-3} \exp \left(\frac{-K^{2}}{2 A_{i}^{2}}\right) \operatorname{erf}\left(\frac{K D_{i}}{A_{i} \Delta_{i} \sqrt{2}}\right) d x \\
= & I_{1}^{(i)}(\alpha, \beta)+I_{2}^{(i)}(\alpha, \beta) .
\end{aligned}
$$

The Rice formula applied to the roots of $T_{i}^{\prime}(x)$ gives

$$
E M^{(i)}(\alpha, \beta)=\int_{\alpha}^{\beta}\left(\frac{\Lambda_{i}}{\pi B_{i}^{2}}\right) d x .
$$

Before we give generic definitions for the individual elements in (2.1) and (2.2), we note two fundamental facts that make these definitions easier,

$$
\operatorname{var}\left\{T_{i}(x)-L\right\}=\operatorname{var}\left\{T_{i}(x)\right\}
$$

and

$$
\operatorname{cov}\left\{T_{i}(x)-L, T_{i}^{\prime}(x)\right\}=\operatorname{cov}\left\{T_{i}(x), T_{i}^{\prime}(x)\right\} .
$$

Using the properties (2.3) and (2.4), we define the variance and covariance elements of (2.1) and (2.2) as

$$
\begin{gathered}
A_{i}^{2}=\operatorname{var}\left\{T_{i}(x)\right\}, \quad B_{i}^{2}=\operatorname{var}\left\{T_{i}^{\prime}(x)\right\}, \quad C_{i}^{2}=\operatorname{var}\left\{T_{i}^{\prime \prime}(x)\right\}, \\
D_{i}=\operatorname{cov}\left\{T_{i}(x), T_{i}^{\prime}(x)\right\}, \quad E_{i}=\operatorname{cov}\left\{T_{i}^{\prime}(x), T_{i}^{\prime \prime}(x)\right\}, \\
\Delta_{i}^{2}=A_{i}^{2} B_{i}^{2}-D_{i}^{2}, \quad \text { and } \quad \Lambda_{i}^{2}=B_{i}^{2} C_{i}^{2}-E_{i}^{2} .
\end{gathered}
$$


To enable us to prove Theorem 2 as simply as possible, we define $Q^{(i)}(\alpha, \beta)$ to be the number of roots of $T_{i}^{\prime \prime}(x)$ (the second derivative of $\left.T_{i}(x)\right)$ on the interval $(\alpha, \beta)$ and we give the Rice formula for the expected number of roots of $T_{i}^{\prime \prime}(x)$.

where

$$
E Q^{(i)}(\alpha, \beta)=\int_{\alpha}^{\beta}\left(\frac{\Psi_{i}}{\pi C_{i}^{2}}\right) d x,
$$

$$
F_{i}^{2}=\operatorname{var}\left\{T_{i}^{(3)}(x)\right\}, \quad G_{i}=\operatorname{cov}\left\{T_{i}(x), T_{i}^{(3)}(x)\right\},
$$

and

$$
\Psi_{i}^{2}=C_{i}^{2} F_{i}^{2}-G_{i}^{2}
$$

3. Evaluation of variances and covariances. Since the coefficients of (1.1) and (1.2) are independent normally distributed random variables with mean zero and variance one,

$$
\begin{aligned}
& A_{2}^{2}=\sum_{j=1}^{n} \sinh ^{2} j x, \\
& B_{1}^{2}=\sum_{j=1}^{n} j^{2} \sinh ^{2} j x, \\
& B_{2}^{2}=\sum_{j=1}^{n} j^{2} \cosh ^{2} j x, \\
& C_{1}^{2}=\sum_{j=1}^{n} j^{4} \cosh ^{2} j x, \\
& C_{2}^{2}=\sum_{j=1}^{n} j^{4} \sinh ^{2} j x, \\
& D_{2}=\sum_{j=1}^{n} j \cosh ^{2} x \sinh j x, \\
& E_{1}=E_{2}=\sum_{j=1}^{n} j^{3} \cosh j x \sinh j x, \\
& F_{1}^{2}=\sum_{j=1}^{n} j^{6} \sinh ^{2} j x .
\end{aligned}
$$

At this point, we rewrite (3.1), (3.2), (3.3), (3.4), (3.6), and (3.7) in a form we utilize later. We observe that

$$
\sinh ^{2} j x=\frac{(\cosh 2 j x-1)}{2},
$$

and use the hyperbolic equivalent to the two formulae given at 1.342 .2 in [6, p. 36], that is

$$
\begin{aligned}
\sum_{j=1}^{n} \cosh 2 j x & =\frac{\cosh (n+1) x \sinh n x+\cosh n x \sinh (n+1) x}{2 \sinh x}-\frac{1}{2} \\
& =\frac{\sinh (2 n+1) x}{2 \sinh x}-\frac{1}{2} .
\end{aligned}
$$


Thus, summing (3.9) and employing (3.10), we find that

$$
A_{2}^{2}=\frac{\sinh (2 n+1) x}{4 \sinh x}-\frac{(2 n+1)}{4} .
$$

By repeated differentiation of (3.11), we find that

$$
\begin{aligned}
B_{i}^{2}= & \left(2 n^{2}+2 n+1\right) \frac{\sinh (2 n+1) x}{8 \sinh x}-(2 n+1) \frac{\cosh x \cosh (2 n+1) x}{8 \sinh ^{2} x} \\
& +\frac{\sinh (2 n+1) x}{8 \sinh ^{3} x}+\frac{(-1)^{i} n(n+1)(2 n+1)}{12}, \quad i=1,2, \\
C_{1}^{2}= & \left(2 n^{4}+4 n^{3}+6 n^{2}+4 n+1\right) \frac{\sinh (2 n+1) x}{8 \sinh x} \\
& -\left(2 n^{2}+2 n+1\right)(2 n+1) \frac{\cosh x \cosh (2 n+1) x}{8 \sinh ^{2} x} \\
& +\left(3 n^{2}+3 n+2\right) \frac{\sinh (2 n+1) x}{4 \sinh 3}-3(2 n+1) \frac{\cosh x \cosh (2 n+1) x}{8 \sinh ^{3} x} \\
& +3 \frac{\sinh ^{3}(2 n+1) x}{8 \sinh x}+\frac{n(n+1)(2 n+1)\left(3 n^{2}+2 n-1\right)}{60}, \\
D_{2}= & (2 n+1) \frac{\cosh (2 n+1) x}{8 \sinh x}-\frac{\cosh x \sinh (2 n+1) x}{8 \sinh x}, \\
E_{1}= & E_{2}=\left(n^{2}+n+1\right)(2 n+1) \frac{\cosh (2 n+1) x}{8 \sinh x} \\
& -\left(3 n^{2}+3 n+1\right) \frac{\cosh x \sinh ^{2}(2 n+1) x}{8 \sinh ^{2} x} \\
& +(6 n+3) \frac{\cosh (2 n+1) x}{16 \sinh x}-\frac{\left.3 \cosh x \sinh ^{3} x+1\right) x}{16 \sinh ^{4} x} .
\end{aligned}
$$

4. Proof of the theorems. Looking at the properties of the random coefficients in (1.1) and (1.2), it is clear that $E N_{k}^{(i)}(0, \infty)=E N_{K}^{(i)}(-\infty, 0)$ and $E M^{(i)}(0, \infty)=E M^{(i)}(-\infty, 0)$. In the proofs that follow, we consider the interval $(0, \infty)$ only, it proves advantageous to break this interval into the three subintervals $(0, \sqrt{\log n} / n),(\sqrt{\log n} / n, 1)$, and $(1, \infty)$. We start by proving Theorem 1 and Theorem 2 on the interval $(0, \sqrt{\log n} / n)$.

From Rolle's theorem, it is evident that, for any differentiable function $P(x)$ with $r$ roots in the interval $(a, b), P^{\prime}(x)$ (the derivative of $\left.P(x)\right)$ has $r^{\prime}$ roots on $(a, b)$, where

$$
r \leq r^{\prime}+1
$$

From (2.1), we know that

$$
E N_{K}^{(2)}(\alpha, \beta) \leq E N_{0}^{(2)}(\alpha, \beta)
$$

where $N_{K}^{(2)}(\alpha, \beta)$ and $N_{0}^{(2)}(\alpha, \beta)$, are the numbers of $K$ level and zero level crossings of (1.2), respectively, on the interval $(\alpha, \beta)$. Employing (4.1), (4.2), (2.2), (3.3), and (3.5), 
we find that

$$
\begin{aligned}
E N_{K}^{(2)}\left(0, \frac{\sqrt{\log n}}{n}\right) & \leq E M^{(2)}\left(0, \frac{\sqrt{\log n}}{n}\right)+1 \leq\left(\frac{1}{\pi}\right) \int_{0}^{\sqrt{\log n} / n}\left(\sqrt{\frac{C_{2}^{2}}{B_{2}^{2}}}\right) d x+1 \\
& \leq\left(\frac{1}{\pi}\right) \int_{0}^{\sqrt{\log n} / n} n d x+1=O(\sqrt{\log n}) .
\end{aligned}
$$

Obviously, taking advantage of the same analysis and using (2.6), (3.4), and (3.8), we can show that

$$
\begin{aligned}
E M^{(1)}\left(0, \frac{\sqrt{\log n}}{n}\right) & \leq E Q^{(1)}\left(0, \frac{\sqrt{\log n}}{n}\right)+1 \\
& \leq\left(\frac{1}{\pi}\right) \int_{0}^{\sqrt{\log n} / n} n d x+1 \\
& =O(\sqrt{\log n}) .
\end{aligned}
$$

We can show that the second integral on the right-hand side of (2.1) does not contribute to the leading behaviour of $E N_{K}^{(2)}(0, \infty)$. Since we know that $\operatorname{erf}(x) \leq \sqrt{\pi / 2}$, for all values of $x$, and by making the simple substitution $u=K / A_{2}$, it is clear that

$$
\begin{aligned}
I_{2}^{(2)}(0, \infty) & \leq\left(\frac{1}{\sqrt{2 \pi}}\right) \int_{0}^{\infty} K D_{2} A_{2}^{-3} \exp \left(\frac{-K^{2}}{2 A_{2}^{2}}\right) d x \\
& =\left(\frac{1}{\sqrt{2 \pi}}\right) \int_{0}^{\infty} \exp \left(\frac{-u^{2}}{2}\right) d u=\frac{1}{2} .
\end{aligned}
$$

Having derived (4.5), it becomes apparent that we can handle the remaining analysis required for both theorems in tandem. At this point, we concentrate on the interval $(\sqrt{\log n} / n, 1)$; this is the only interval that contributes to the leading behaviour of $E N_{K}^{(2)}(0, \infty)$ and $E M^{(1)}(0, \infty)$. To find the dominant terms in (3.11), (3.12), (3.13), (3.14), and (3.15), we observe that, in this interval,

$$
\operatorname{coth} x<\frac{e}{x}<\frac{e n}{\sqrt{\log n}}
$$

Employing (4.6), it becomes a trivial task to show that the derivative of $f_{n, p}(x)=$ $\sinh n x /(\sinh x)^{p}$ is positive for all $p<e^{-1} \sqrt{\log n}$. Therefore, since $\sinh (x)<4 x$ in $(0,1)$,

$$
f_{n, p}(x) \geq \sinh \{\sqrt{\log n}\}\left[n(4 \sqrt{\log n})^{-1}\right]^{p}
$$

for all sufficiently large $n$.

Hence,

$$
f_{2 n+1, p}(x) \geq\left(\frac{n^{p}}{48}\right)(\log n)^{-p / 2} \exp \{\sqrt{\log n}\}
$$

for all $p=1,2,3,4, \ldots$ Now, using (3.11), (3.12), (3.13), (3.14), and (3.15) and since $\sinh x \geq x / 4$ for all $x \in(0,1)$, we can show that, for all sufficiently large $n$, 


$$
\begin{aligned}
& A_{2}^{2}=\frac{\sinh (2 n+1) x}{4 \sinh x}\left\{1+O\left\{\frac{\sqrt{\log n}}{\exp (\sqrt{\log n})}\right\},\right. \\
& B_{1}^{2}=B_{2}^{2}=\left(2 n^{2}+2 n+1\right) \frac{\sinh (2 n+1) x}{8 \sinh x}\left\{1+O\left\{\frac{1}{\sqrt{\log n}}\right\}\right\}, \\
& C_{1}^{2}=\left(2 n^{4}+4 n^{3}+6 n^{2}+4 n+1\right) \frac{\sinh (2 n+1) x}{8 \sinh x}\left\{1+O\left\{\frac{1}{\sqrt{\log n}}\right\}\right\}, \\
& D_{2}=(2 n+1) \frac{\cosh (2 n+1) x}{8 \sinh x}\left\{1+O\left\{\frac{1}{\sqrt{\log n}}\right\}\right\}, \\
& E_{1}=E_{2}=\left(n^{2}+n+1\right)(2 n+1) \frac{\cosh (2 n+1) x}{8 \sinh x}\left\{1+O\left\{\frac{1}{\sqrt{\log n}}\right\}\right\} .
\end{aligned}
$$

Due to cancellation of terms, it is not sufficient to use (4.9), (4.10), and (4.12) to compute $\Delta_{2}^{2}$. Instead, we use (3.11), (3.12), and (3.14) to show that

$$
\Delta_{2}^{2}=\frac{\sinh ^{2}(2 n+1) x}{64 \sinh ^{4} x}\left\{1+O\left\{\frac{1}{\sqrt{\log n}}\right\}\right\}
$$

We compute $\Psi_{1}^{2}$ from (4.10), (4.11), and (4.13) directly to give

$$
\Psi_{1}^{2}=n^{4} \frac{\sinh ^{2}(2 n+1) x}{64 \sinh ^{4} x}\left\{1+O\left\{\frac{1}{\sqrt{\log n}}\right\}\right\} .
$$

Since we have already determined $I_{2}^{(2)}(0, \infty)$, we concentrate on $I_{1}^{(2)}(\sqrt{\log n} / n, 1)$. From (2.1), (4.9), (4.10), and (4.12), we have

$$
\begin{aligned}
I_{1}^{(2)}\left(\frac{\sqrt{\log n}}{n}, 1\right)= & (2 \pi)^{-1} \int_{\sqrt{\log n} / n}^{1} \operatorname{cosech} x\left\{1+O\left\{\frac{1}{\sqrt{\log n}}\right\}\right\} d x \\
& \times \exp \left(-8 n^{2} \frac{\sinh ^{3} x K^{2}}{\sinh (2 n+1) x}\right) d x \\
= & (2 \pi)^{-1} \int_{\sqrt{\log n} / n}^{1} \operatorname{cosech} x\left\{1+O\left\{\frac{1}{\sqrt{\log n}}\right\}\right\} d x \\
& +O\left(K^{2} n^{2} \int_{\sqrt{\log n} / n}^{1} \frac{\sinh ^{2} x}{\sinh (2 n+1) x} d x\right) .
\end{aligned}
$$

The first integral on the right-hand side of (4.16) can be evaluated directly. To determine the second integral, we use the fact that $x \leq \sinh x \leq 4 x$ on the interval $0 \leq x \leq 1$. Thus,

$$
\begin{aligned}
I_{1}^{(2)}\left(\frac{\sqrt{\log n}}{n}, 1\right)= & (2 \pi)^{-1}\left\{\log \left(\tanh \left(\frac{1}{2}\right)\right)-\log \left(\tanh \left(\frac{\sqrt{\log n}}{2 n}\right)\right)\right\} \\
& +O\left\{K^{2} n \int_{2 \sqrt{\log n}}^{2 n} u^{2} \operatorname{cosech}(u) d u\right\} \\
= & (2 \pi)^{-1} \log n+O(\log \log n)+O\left(\frac{K^{2}}{n}\right) .
\end{aligned}
$$


Since $K=o(\sqrt{n \log n})$, it is clear from (4.17) that $I_{2}^{(2)}(\sqrt{\log n} / n, 1)=(2 \pi)^{-1} \log n$. We now evaluate $E M^{(1)}(\sqrt{\log n} / n, 1)$ using (2.2), (4.10), and (4.15).

$$
\begin{aligned}
E M^{(1)}\left(\frac{\sqrt{\log n}}{n}, 1\right) & =(2 \pi)^{-1} \int_{\sqrt{\log n} / n}^{1} \operatorname{cosech} x d x\left\{1+O\left\{\frac{1}{\sqrt{\log n}}\right\}\right\} \\
& =(2 \pi)^{-1}\left\{\log \left(\tanh \left(\frac{1}{2}\right)\right)-\log \left[\tanh \left\{\frac{\sqrt{\log n}}{2 n}\right\}\right]\right\} \\
& =(2 \pi)^{-1} \log n+O\{\log \log n\} .
\end{aligned}
$$

All that remains to complete the proofs of the two theorems is to evaluate $E N_{K}^{(2)}(1, \infty)$ and $E M^{(1)}(1, \infty)$. The same function $f_{n, p}(x)$, as used in the previous section, is obviously strictly increasing on the interval $(1, \infty)$. Since $\sinh n x>\exp (n x) / 3$ and $\sinh x \leq$ $\exp (x) / 2$, it is clear that

$$
f_{n, p}(x) \geq 2 \frac{\exp (n-p)}{3} .
$$

Using inequality (4.19), it can be shown that

$$
\begin{aligned}
& A_{2}^{2}=\frac{\sinh (2 n+1) x}{4 \sinh x}\{1+O\{n \exp (-2 n)\}\} \\
& B_{1}^{2}=B_{2}^{2}=\left(2 n^{2}+2 n+1\right) \frac{\sinh (2 n+1) x}{8 \sinh x}\left\{1+O\left\{\frac{1}{n}\right\}\right\} \\
& C_{1}^{2}=\left(2 n^{4}+4 n^{3}+6 n^{2}+4 n+1\right) \frac{\sinh (2 n+1) x}{8 \sinh x}\left\{1+O\left\{\frac{1}{n}\right\}\right\} \\
& D_{2}=(2 n+1) \frac{\cosh (2 n+1) x}{8 \sinh x}\left\{1+O\left\{\frac{1}{n}\right\}\right\} \\
& E_{1}=E_{2}=\left(n^{2}+n+1\right)(2 n+1) \frac{\cosh (2 n+1) x}{8 \sinh x}\left\{1+O\left\{\frac{1}{n}\right\}\right\} \\
& \Delta_{2}^{2}=\frac{\sinh ^{2}(2 n+1) x}{64 \sinh ^{4} x}\left\{1+O\left\{\frac{1}{n}\right\}\right\} \\
& \Lambda_{1}^{2}=n^{4} \frac{\sinh ^{2}(2 n+1) x}{64 \sinh ^{4} x}\left\{1+O\left\{\frac{1}{n}\right\}\right\}
\end{aligned}
$$

Employing (2.1), (4.2), (4.20), and (4.25), we find that

$$
\begin{aligned}
E N_{K}^{(2)}(1, \infty) & \leq\left(\frac{1}{2 \pi}\right) \int_{1}^{\infty} \operatorname{cosech} x d x\left\{1+O\left\{\frac{1}{n}\right\}\right\} \\
& =\left.\left(\frac{1}{2 \pi}\right) \log \left[\tanh \left(\frac{x}{2}\right)\right]\right|_{1} ^{\infty}\left\{1+O\left\{\frac{1}{n}\right\}\right\} \\
& =O\{1\} .
\end{aligned}
$$

Similarly, using (2.2), (4.21), and (4.26), we find that

$$
E M^{(1)}(1, \infty)=O\{1\} .
$$


If we combine (4.3), (4.5), (4.16), and (4.27), it is obvious that Theorem 1 is proved. Bringing together (4.4), (4.18), and (4.28), the proof of the second theorem is also complete.

\section{REFERENCES}

[1] A. T. Bharucha-Reid and M. Sambandham, Random Polynomials, Probability and Mathematical Statistics, Academic Press, Inc., Orlando, Fla., New York, 1986. MR 87m:60118. Zbl 615.60058.

[2] M. Das, On the real zeros of a random polynomial with hyperbolic elements, Unpublished Ph.D. Dissertation, India, 1971.

[3] K. Farahmand, Real zeros of random algebraic polynomials, Proc. Amer. Math. Soc. 113 (1991), no. 4, 1077-1084. MR 92c:60080. Zbl 734.60059.

[4] L_ Level crossings of a random polynomial with hyperbolic elements, Proc. Amer. Math. Soc. 123 (1995), no. 6, 1887-1892. MR 95g:60082. Zbl 822.60047.

[5] K. Farahmand and M. Jahangiri, On real zeros of random polynomials with hyperbolic elements, Internat. J. Math. Math. Sci. 21 (1998), no. 2, 347-350. Zbl 908.60042.

[6] I. S. Gradshteyn and I. M. Ryzhik, Table of Integrals, Series, and Products, 5th ed., Academic Press, Inc., Boston, New York, London, 1994. MR 94g:00008. Zbl 940.06297.

[7] J. E. Littlewood and A. C. Offord, On the number of real roots of a random algebraic equation, J. London Math. Soc. 13 (1938), 288-295. Zbl 020.13604.

[8] On On the number of real of a random algebraic equation II, Math. Proc. Cambridge Philos. Soc. 35 (1939), 133-148. Zbl 021.03702.

[9] A. C. Offord, The distribution of the values of an entire function whose coefficients are independent random variables. II, Math. Proc. Cambridge Philos. Soc. 118 (1995), no. 3, 527-542. MR 96m:30046. Zbl 846.60037.

[10] S. O. Rice, Mathematical analysis of random noise, Bell System Tech. J. 24 (1945), 46-156. MR 6,233i. Zbl 063.06487.

[11] J. E. Wilkins, Jr., An asymptotic expansion for the expected number of real zeros of a random polynomial, Proc. Amer. Math. Soc. 103 (1988), no. 4, 1249-1258. MR 90f:60105. Zbl 656.60062.

FARAHMAND AND HANNIGAN: DEPARTMENT OF MATHEMATICS, UNIVERSITY OF UlSTER, JORDASTOWN, CO. ANTRIM BT37 OQB, UK 


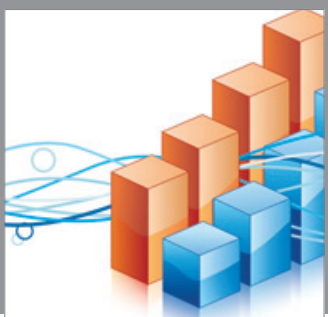

Advances in

Operations Research

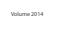

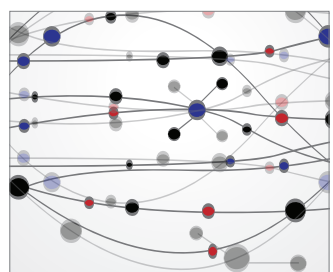

\section{The Scientific} World Journal
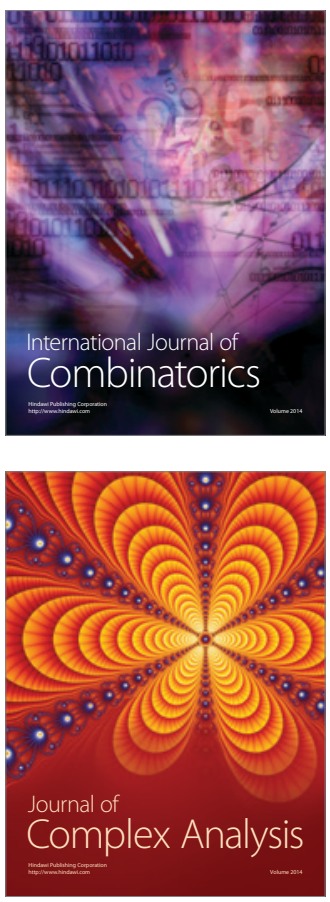

International Journal of

Mathematics and

Mathematical

Sciences
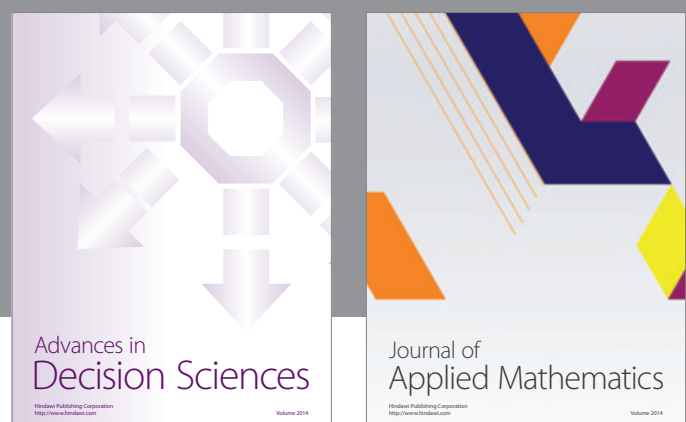

Journal of

Applied Mathematics
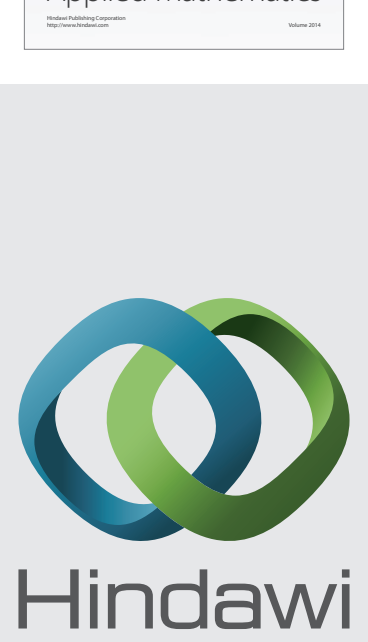

Submit your manuscripts at http://www.hindawi.com
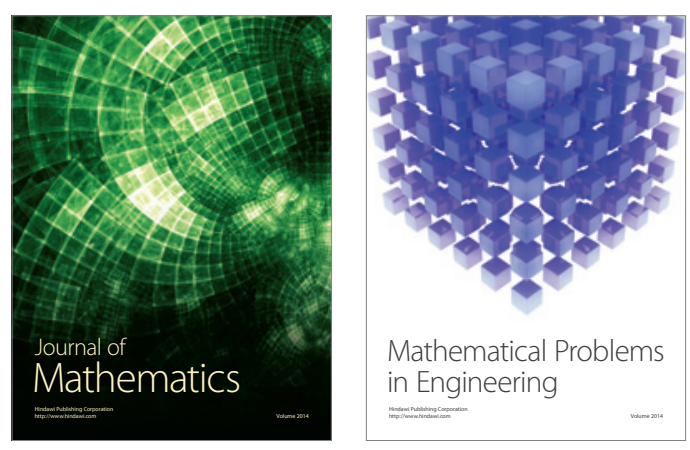

Mathematical Problems in Engineering
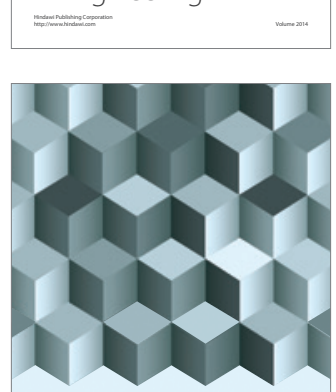

Journal of

Function Spaces
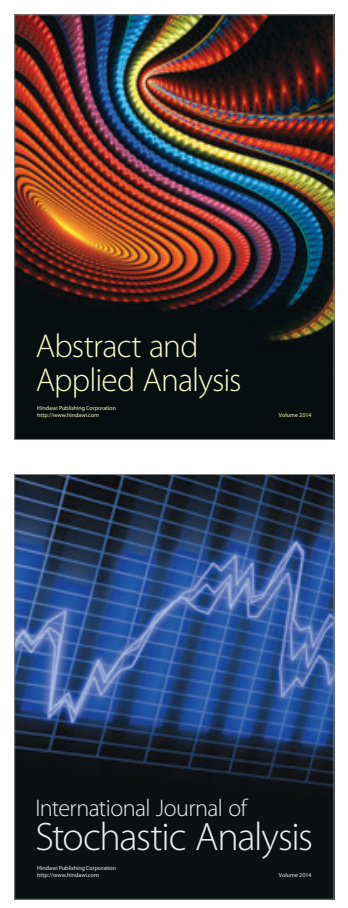

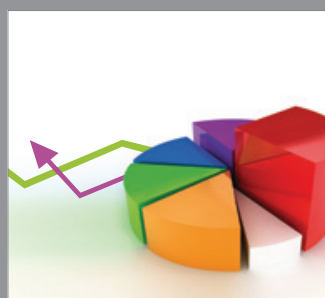

ournal of

Probability and Statistics

Promensencen
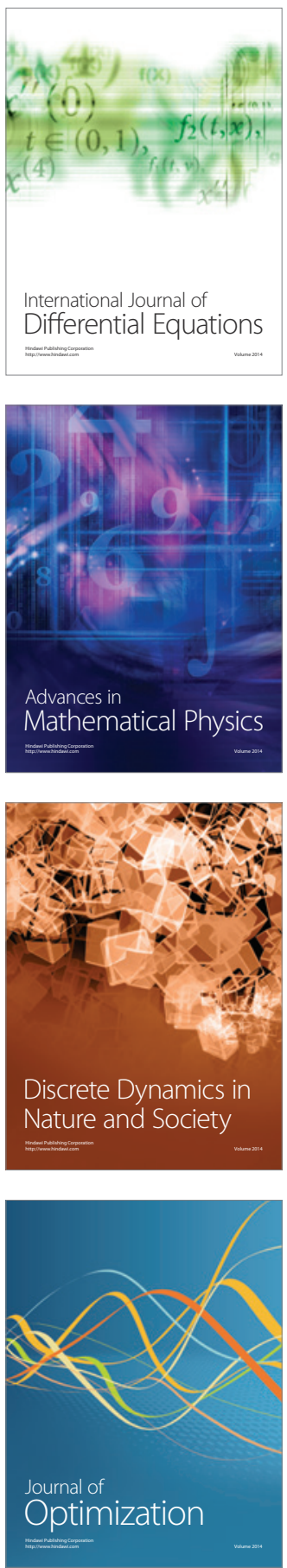